\title{
Harmonic Suppression Method Based on Immune Particle Swarm Optimization Algorithm in Micro-Grid
}

\author{
Ying Zhang, Yufeng Gong, Junyu Chen, Jing Wang \\ Department of Information Engineering, Zhejiang University of Technology, Hangzhou, China \\ Email: zizaifeihua666@163.com
}

Received January 2014

\begin{abstract}
Distributed generation has attracted great attention in recent years, thanks to the progress in new-generation technologies and advanced power electronics. And micro-grid can make full use of distributed generation, so it has been widespread concern. On the other hand due to the extensive use of power electronic devices and many of the loads within micro-grid are nonlinear in nature, Micro-grid generate a large number of harmonics, so harmonics pollution needs to be addressed. Usually we use passive filter to filter out harmonic, in this paper, we propose a new method to optimize the filter parameters, so passive filter can filter out harmonic better. This method utilizes immune particle swarm optimization algorithm to optimize filter parameters. It can be shown from the simulation results that the proposed method is effective for micro-grid voltage harmonics compensation.
\end{abstract}

\section{Keywords}

Micro-Grid; Immune Particle Swarm Optimization Algorithm; Harmonic Compensation

\section{Introduction}

New energy and renewable energy become the current research focus; micro-grid combines the advantages of distributed generation technologies, resulting in widespread attention [1]. Micro-grid supports the power grid effectively; micro-grid has two kinds of operation mode islanding mode and grid-connected mode [2]. The non-linear load because of operation of inverters and other power electronic equipments which is called harmonic has a serious impact on normal operation of Inverters and other power electronic equipments. Also harmonic current will cause the temperature of the equipment to rise, which directly affects the service life of equipments [3]. Currently there are many methods of micro-grid harmonic suppression which are divided into three categories. The first category is to suppress harmonics essentially, including active mode (creating inverters which only produce small harmonics) and passive mode (installing filters in the vicinity of the harmonics source); The second category is the application of instantaneous reactive power theory, but the theory is not mature. The third category is the integration of intelligent neural network and harmonic suppression, but the effect is not ideal when the harmonic variation is great [4]. In the first category techniques, installing the filter is the 
most common means which is the most effective and also suitable for universal application. Among them, the passive filter (LC filter) has a simple structure with low cost and high operational reliability, so it is a widely used harmonic treatment method [5,6].

Currently, there are many methods of optimizing LC filter parameters in micro-grid system, which can be summed up into two categories: One is the conventional optimization methods including nonlinear programming method, linear programming method and interior point method; the other is artificial intelligence optimization algorithm, including genetic algorithm, particle swarm optimization algorithm, and a variety of evolutionary programming methods. The first method's merit is rapid calculation and reliable, but first method requires some assumptions, such as continuous, derivable and a single peak and so on. In solving some problems, it may need to treat integer variables as continuous variables, and then get the optimal solution so as to normalize to the whole. But for some large-scale actual system, the error generated by normalizing to the whole is generally unacceptable. Genetic algorithm for optimization is most widely used in the second method, it has no continuity or derivable requirements, it just needs an adaptive function or performance indicators, its main drawbacks are "premature convergence" issues and the convergence rate which is difficult to meet the needs of real-time control [7]. Particle Swarm Optimization (PSO) is a global stochastic optimization algorithm proposed by American scholars Kennedy and Eberhart in 1995 [8], and as a new intelligent computing method based on group, it shows strong advantages in solving problems. Compared with other evolutionary algorithms, it is easy to implement, and it has less adjustable parameters, therefore it is suitable for micro-grid system to optimize the filter parameters. However, its disadvantage is easy to fall into local extreme point.

Immune algorithm simulates biological immune principle, it utilizes the diversity of immune system antibody and self-regulatory function to maintain the diversity of population [9], and therefore it overcomes the premature in the optimization process to ensure that the system converges to the global optimal solution quickly. In [10], the immune algorithm and particle swarm algorithm is combined so as to propose immune particle swarm optimization (IPSO). In this paper, the immune particle swarm algorithm is applied to optimize filter parameter in micro-grid and suppress harmonic generation in micro-grid.

\section{Model of Harmonic Suppression in Micro-Grid}

\subsection{The Model of Micro-Grid System}

Micro-grid is connected together by voltage source inverter. A three-leg VSI with an LC filter with a coupling inductor form the power circuit, whereas three control loops form the control structure. Specifically, a power sharing controller is used to generate the magnitude and frequency of the fundamental output voltage of the inverter according to the droop characteristic, by emulating the operation of a conventional synchronous generator; a voltage controller is used to synthesize the reference filter-inductor current vector [11]; and a current controller is adopted to generate the command voltage vector to be synthesized by a space vector pulse width modulation (SVPWM) module. The output voltage of the inverter includes not only the sinusoidal signal of 50HZ, but also various harmonics, so it need to be filtered and then can be provided to the load.

\subsection{The Objective Function}

In the state of islanding or grid-connected, we usually use voltage harmonic ratio of load as the standard of evaluating the merits of filter parameters. Voltage harmonic distortion rate can calculated by the RMS of fundamental and harmonic voltage, where RMS of voltage getting from the Fourier transforming of matlab programming. Therefore, the voltage harmonic distortion called THD can be obtained by Equation (1),

$$
T H D=\frac{\sqrt{\left(U_{2} * U_{2}+U_{3} * U_{3}+\cdots+U_{n} * U_{n}\right)}}{U_{1}}
$$

where THD is the voltage harmonic distortion, $U_{n}$ is RMS of the n-th harmonic voltage, $U_{1}$ is the RMS of fundamental voltage.

\subsection{Constraints Condition}

General principles of the LC filter design can be shown as follows: 


$$
\begin{gathered}
10 f_{n} \leq f_{c} \leq f_{s} / 10 \\
f_{c}=1 /\left(2 \pi \sqrt{L_{f} C_{f}}\right)
\end{gathered}
$$

where $f_{c}$ represents the resonant frequency of LC filter; $f_{n}$ represents modulation wave frequency, $f_{s}$ represents carrier signal frequency of SPWM.

\section{Immune Particle Swarm Algorithm}

\subsection{Particle Swarm Algorithm}

In the particle swarm optimization, the solutions of the problem are seen as particles in a search space without size and weight. Each of particles motions in the solution space, and one speed determines its direction and distance. The particles find the optimal solutions in iterations, in the every iteration, particles tracking two extremes: the optimal solution pbest which is found by the particles themselves so far and the optimal solution gbest which is found by populations so far. Velocity and position of Particles are updated by the following two formulas.

$$
\begin{gathered}
v_{i d}^{t+1}=w \times v_{i d}^{t}+c_{1} \times r_{1} \times\left(p_{i d}^{t}-x_{i d}^{t}\right)+c_{2} \times r_{2} \times\left(g_{i d}^{t}-x_{i d}^{t}\right) \\
x_{i d}^{t+1}=x_{i d}^{t}+v_{i d}^{t+1}
\end{gathered}
$$

where $d=1,2, \cdots, D$ is the dimension of the target search space, $i=1,2, \cdots, N$ is the number of particles, $v_{i d}^{t}$ is the velocity of d-dimensional of particle $i$ at the t-th iteration, $x_{i d}^{t}$ is the position of d-dimensional of particle $i$ at the $\mathrm{t}$-th iteration, $p_{\text {id }}^{t}$ is individual optimal position of d-dimensional of particle $i$ at the $\mathrm{t}$-th iteration, also denoted as pbest; $g_{i d}^{t}$ is global optimal position of d-dimensional of particle $i$ at the t-th iteration, also denoted as gbest; $r_{1}$ and $r_{2}$ are the random numbers uniformly distributed among [0,1]; as the acceleration factors, $c_{1}$ and $c_{2}$ are used to adjust the step of each iteration, $\omega$ is the inertia factor .

\subsection{Immune Particle Swarm Algorithm}

To overcome the problem of premature convergence of particle swarm algorithm, and to improve the global search capability, the principles of the immune mechanisms of the immune system are introduced into particle swarm algorithm, which is very helpful to improve the ability of the global convergence of particle swarm algorithm. Immune particle swarm algorithm is an improved particle swarm algorithm based on biological immune mechanism. The objective function of the problem that needs to be solved corresponds to the antigen which invade into the biological life and the solution of the problem corresponds to antibodies which is produced in the immune system [12].

After some successive iterations of PSO, if there are no better individuals, then the particle swarm algorithm has been caught in a local optimum, so we need to select a certain number of individuals according to the antibody concentration to be replaced with randomly generated individuals, in order to maintain the population diversity and avoid local optima. The individual with lower adaptation value and higher concentration will have higher probability of being replaced, [13] gives the probability that i-th individual being replaced. Definitions are as follows:

The definitions of the probability of individual being replaced: the replacement probability of the i-th individual called $R_{i}$ is determined by the concentration probability of the individual $R_{i c}$ and the fitness probability of the(M-i)-th individual called $R_{(\mathrm{M}-\mathrm{i}) \text { f. }}$ ( $M$ is the size of the population), specifically:

$$
R_{i}=\alpha R_{(m-i) f}+(1-\alpha) R_{i c} \quad 0 \leq \alpha \leq 1
$$

where $f_{i}$ is the fitness value of individual $i, R_{i c}=\frac{m}{M}, m$ is the total number of individuals which is less than a fixed value distance with i-th individual.

\subsection{Algorithm Steps}

Steps of immune particle swarm optimization algorithm are as follows:

1) Initialize parameters; 
2) Initialize the position and velocity of the particles and the fitness value;

3) Update the position and velocity of the particles according to formula (4) and (5);

4) Update the fitness value of the particles called present;

5) Evaluation of fitness, update individual historical extremum called pbest;

6) Update the global extremum called gbest;

7) If the results meet the accuracy requirements then get out of the loop, otherwise skip to step 8);

8) Use immune mechanism, when there are no obviously superior individuals in the population after the DS-th iteration. Calculate concentration of the i-th individual and fitnesss probability called $R_{(\mathrm{M}-\mathrm{i}) \mathrm{f}}$ of the (M-i)-th individual, then obtain replacement rate of the i-th individual. If the replacement probability of individual is greater than predetermined probability value, then the particle is replaced by particle which is randomly generated. Skip to step 3).

\section{Application of Immune Particle Swarm Algorithm on Harmonic Suppression in Micro-Grid}

\subsection{Invocation of Immune Particle Swarm Algorithm on Micro-Grid Simulation System}

At present, when immune particle swarm optimization in the micro-grid system tunes the filter parameters, we need to know the exact relationship between filter parameters and the objective function, so we should transform micro-grid system into state equation or transfer function, and then run the IPSO program to get optimal value of the filter parameters. However, in micro-grid with complex internal system, state equation or transfer function is difficult to be obtained directly, so it requires much more time to get state equation or transfer function of micro-grid, thereby it increases computation, and even affects optimize efficiency.

This paper presents a new approach to solve this problem, when we use IPSO program to optimize the filter parameters of micro-grid system, only need to know the filter parameters which are needed to be optimized and the objective function, then write micro-grid simulation system program called fitness. In detail, firstly, open the micro-grid simulation system with the function of open_system and set LC filter parameters which are needed to be optimized as variables with the function of set_param, secondly, use the function of sim to control the operation of the micro-grid simulation system, thirdly, write the objective function program. Then call micro-grid simulation system program fitness with IPSO program. When IPSO program gets filter parameter values, micro-grid system will run to get the value of the objective function. When we use IPSO to optimize filter parameters of micro-grid system, we just only to know filter parameters of micro-grid which needed to be optimized and the objective function as merits of the evaluation parameters. Therefore, this method can effectively reduce the amount of calculation, and improve the efficiency of using IPSO to optimize filter parameters of the micro-grid system.

\subsection{Processing Constraints}

When IPSO optimize the filter parameters of micro-grid system, $\mathrm{L}$ and $\mathrm{C}$ are the parameters which need to be optimized. Because the system has two micro-sources, there are a total of four parameters $L 1, L 2, C 1, C 2$ need to be optimized. From the formula (2), (3), we know that there is relationship between $L 1$ and $C 1$ or $L 2$ and $C 2$, and the resonance frequency of the filter $f_{c}$, and the modulation frequency $f_{n}$ and the carrier signal frequency of SVPWM $f_{s}$ are all known, so $C 1$ can be expressed by $L 1$ and $C 2$ can be expressed by $L 2$. It can be seen we do not need additional constraints in the immune particle swarm algorithm, and only use the function of set_param to represent $C 1$ by $L 1$, also $C 2$ can be represented by $L 2$.To some extent, it simplify the program of immune particle swarm algorithm.

\subsection{Steps of Immune Particle Swarm Algorithm Optimizing Filter Parameters of Micro-Grid System}

The steps of Immune particle swarm algorithm optimizing filter parameters of micro-grid system are as follows:

1) Initialize parameters, including the number of the maximum iterations, the number of particles, the number of dimensions, learning factor, inertia weight, the value of the minimum distance between particles and probability of immune replacement;

2) Initialize the position and velocity of the particles and the fitness value, call program of micro-grid simula- 
tion system fitness

3) Update the position and velocity of the particle according to formula (4) and (5);

4) Call program of micro-grid simulation system fitness, and update the fitness value of particles called Present;

5) Evaluation of fitness, update individual historical extremum called pbest and global extremum called gbest;

6) If the results meet the accuracy requirements then get out of the loop, otherwise skip to step 7);

7) Use immune mechanism, when there are no obviously superior individuals in the population after the DS-th iteration. Calculate concentration of the i-th individual and fitnesss probability called $R_{(M-i) f}$ of the (M-i)-th individual according to equation 6), then obtain replacement rate of the i-th individual. If the replacement probability of individual is greater than predetermined probability value, then the particle is replaced by particle which is randomly generated. Skip to step 3.

Specific steps of preparing the program of objective function fitness are as follows:

a) Open the micro-grid simulation system with the function of open_system;

b) Set LC filter parameters of LC filter in the micro-grid system which is needed to be optimized as variables denoted $l$ and $l l$ with the function of set_param;

c) Use the function of sim to control the operation of the micro-grid simulation system;

d) Based on filter parameters which be optimized determine the objective function and write program of the objective function in the micro-grid, also referred to a variable called THD.

\subsection{Flowchart of Immune Particle Swarm Algorithm Optimizing Filter Parameters of Micro-Grid System}

Flowchart of immune particle swarm algorithm optimize filter parameters of micro-grid system is shown in Figure 1.

\section{Simulation Results}

\subsection{Micro-Grid Data and Initial Parameter}

Micro-grid simulation system includes two micro-sources, a power grid. Filter resonance frequency $f_{c}$ is $500 \mathrm{HZ}$, the modulation frequency $f_{n}$ is $50 \mathrm{HZ}$ and SPWM carrier signal frequency $f_{s}$ is $5000 \mathrm{HZ}$. Initial parameters: maximum number of iterations is 100; the number of the initial population of 100; the dimension number is 2; the learning factor $c 1$ and $c 2$ are 1.05; the maximum inertia weight is 1.5 , the minimum value is 0.6 ; the accuracy of the harmonic ratio is $0.05, D S$ is 50 , probability of replacement is 0.6 , the minimum distance between the particles is $1 \mathrm{e}-015$.

\subsection{Simulation Results}

\subsubsection{Case 1}

Micro-grid operates with grid initially and simulation time is $0.1 \mathrm{~s}$. Power grid and two micro-sources provide power to the load together; one micro-source disconnects with the load at $0.05 \mathrm{~s}$, and disconnects the grid at $0.07 \mathrm{~s}$, so micro-grid gets into the island state. $L 1$ is $1.8042 \mathrm{e}-005, L 2$ is $1.6173 \mathrm{e}-005$, and the harmonic ratio is 0.0524 by using PSO algorithm to optimize the filter parameters. L1 is $1.3642 \mathrm{e}-5$, L2 is $1.4073 \mathrm{e}-5 \mathrm{and}$ the harmonic ratio is 0.0379 by using IPSO algorithm to optimize the filter parameters. The comparison of $\mathrm{A}$, B, C three-phase voltage of the simulation results by using IPSO to optimize the filter parameters and A, B, C three-phase voltage of the simulation results by using PSO to optimize the filter parameters is shown in Figure 2.

\subsubsection{Case 2}

Micro-grid operates islanding state and simulation time is $0.1 \mathrm{~s}$, only one micro-source provides power to the load, one micro-source connects with the load at $0.05 \mathrm{~s}$, then connect the grid at $0.07 \mathrm{~s}$, so Micro-grid operates with grid-connected. $L 1$ is $1.4042 \mathrm{e}-5, L 2$ is $1.4925 \mathrm{e}-5$, and the harmonic ratio is 0.0596 by using PSO algorithm to optimize the filter parameters. $L 1$ is $1.2368 \mathrm{e}-005, L 2$ is $1.3010 \mathrm{e}-005$ and the harmonic ratio is 0.0497 by using IPSO algorithm to optimize the filter parameters. The comparison of A, B, C three-phase voltage of the simulation results by using IPSO to optimize the filter parameters and A, B, C three-phase voltage of the simulation 


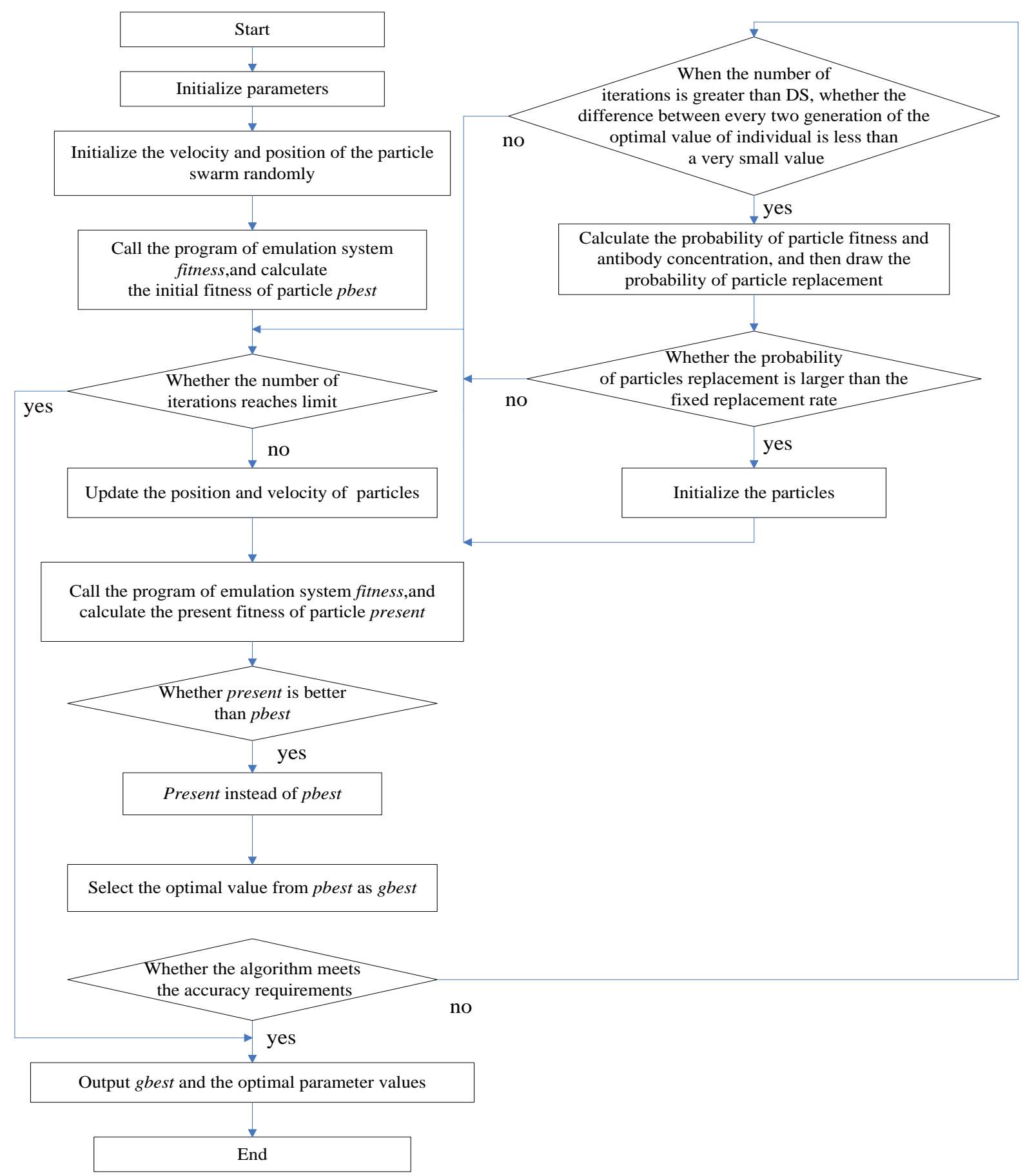

Figure 1. Flowchart of immune particle swarm algorithm optimizing filter parameters of micro-grid system.

results by using PSO to optimize the filter parameters is shown in Figure 3.

\subsubsection{Case 3}

Initially there are only one micro-source and power grid to provide power to load and simulation time is $0.1 \mathrm{~s}$. Then disconnect the power grid at $0.05 \mathrm{~s}$. $L$ is $1.2468 \mathrm{e}-5$, and the harmonic ratio is 0.0605 by using PSO algorithm to optimize the filter parameters. $L$ is $1.1259 \mathrm{e}-005$ and the harmonic ratio is 0.0508 by using IPSO algorithm to optimize the filter parameters. The comparison of A, B, C three-phase voltage of the simulation 


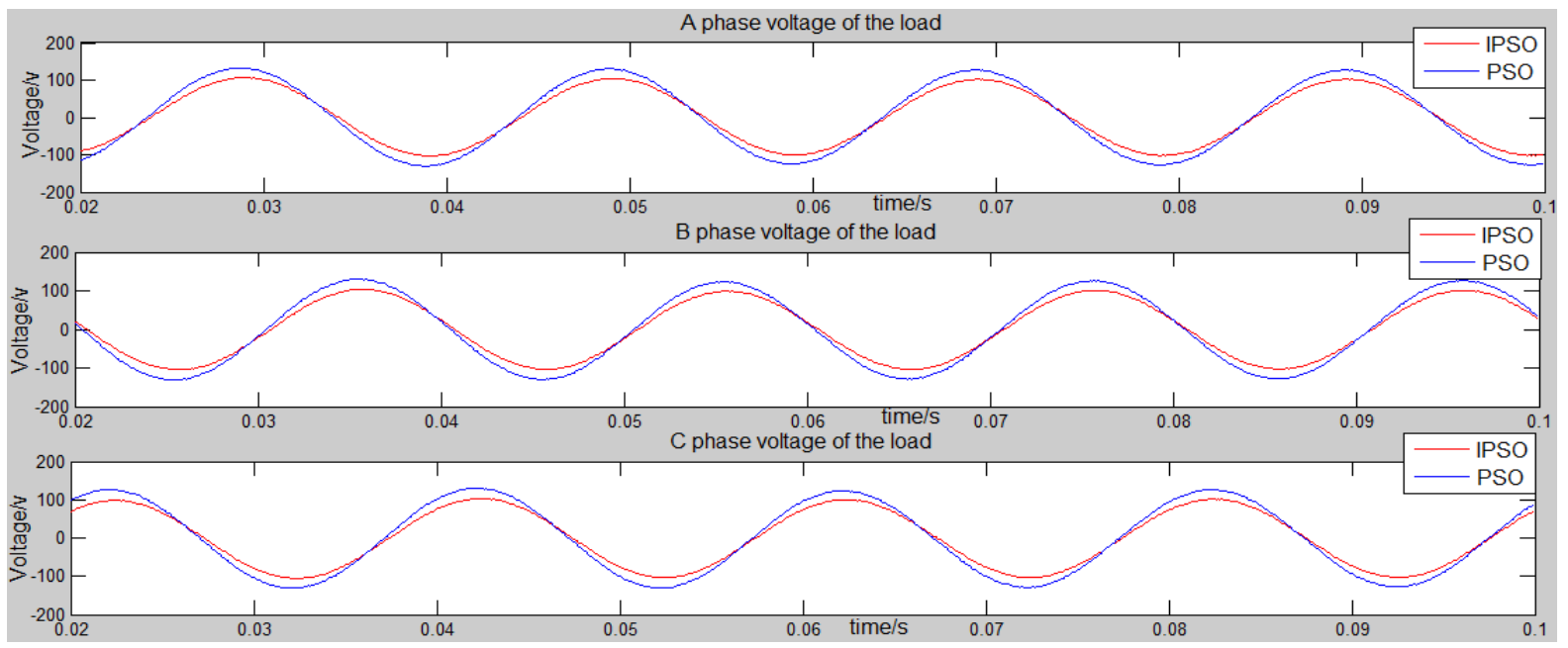

Figure 2. The load output voltage comparison chart of IPSO and PSO optimization from Grid -connected to islanding operation with two micro-sources.

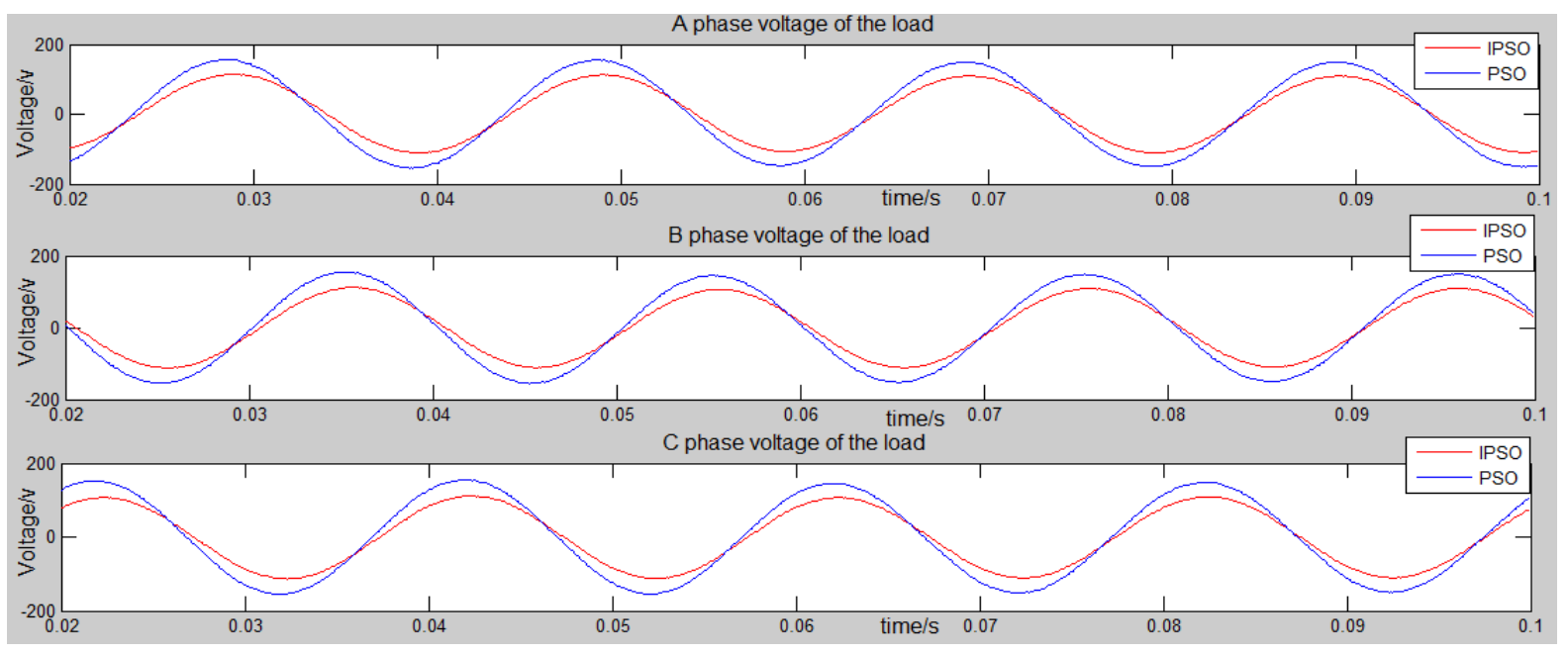

Figure 3. The load output voltage comparison chart of IPSO and PSO optimization from islanding to Grid-connected operation with two micro-sources.

results by using IPSO to optimize the filter parameters and A, B, C three-phase voltage of the simulation results by using PSO to optimize the filter parameters is shown in Figure 4.

\subsubsection{Case 4}

Initially there is only one micro-source to provide power to load and simulation time is $0.1 \mathrm{~s}$. Then connect the power grid with micro-grid at $0.05 \mathrm{~s}$. $L$ is $1.2582 \mathrm{e}-005$, and the harmonic ratio is 0.0616 by using PSO algorithm to optimize the filter parameters. $L$ is $1.1158 \mathrm{e}-005$ and the harmonic ratio is 0.0510 by using IPSO algorithm to optimize the filter parameters. The comparison of A, B, C three-phase voltage of the simulation results by using IPSO to optimize the filter parameters and A, B, C three-phase voltage of the simulation results by using PSO to optimize filter parameters is shown in Figure 5.

Above the cases, according to the Figure 2 of the comparison of A, B, C three-phase voltage of the simulation results by using IPSO to optimize the filter parameters and A, B, C three-phase voltage of the simulation results by using PSO, we can find that the load side output voltage harmonic rate decreases from $5.24 \%$ to $3.79 \%$. And according to the figure 4 of the comparison of A, B, C three-phase voltage of the simulation results by using IPSO and A, B, C three-phase voltage of the simulation results by using PSO, we can find that the load side output voltage harmonic rate decreases from $6.05 \%$ to $5.08 \%$. Therefore, using IPSO to optimize 


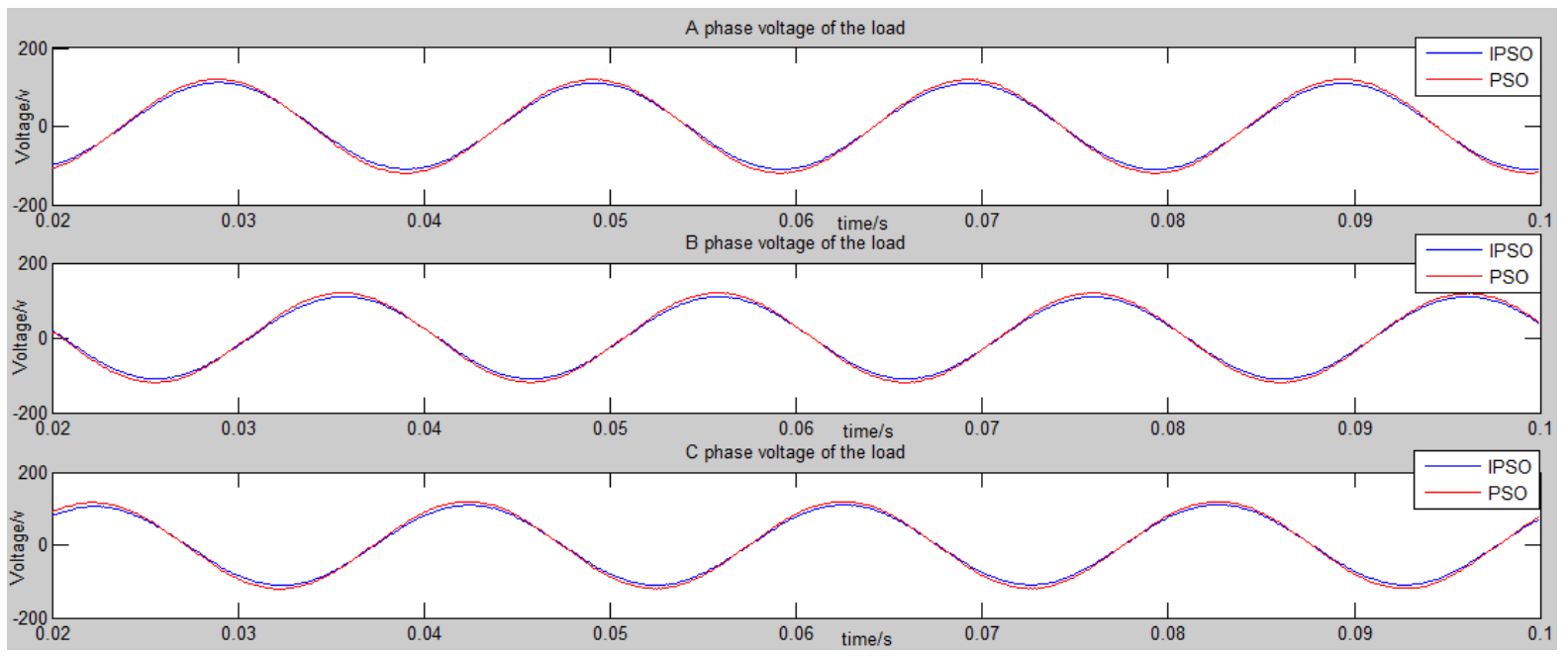

Figure 4. The load output voltage comparison chart of IPSO and PSO optimization from Grid-connected to islanding operation with one micro-source.

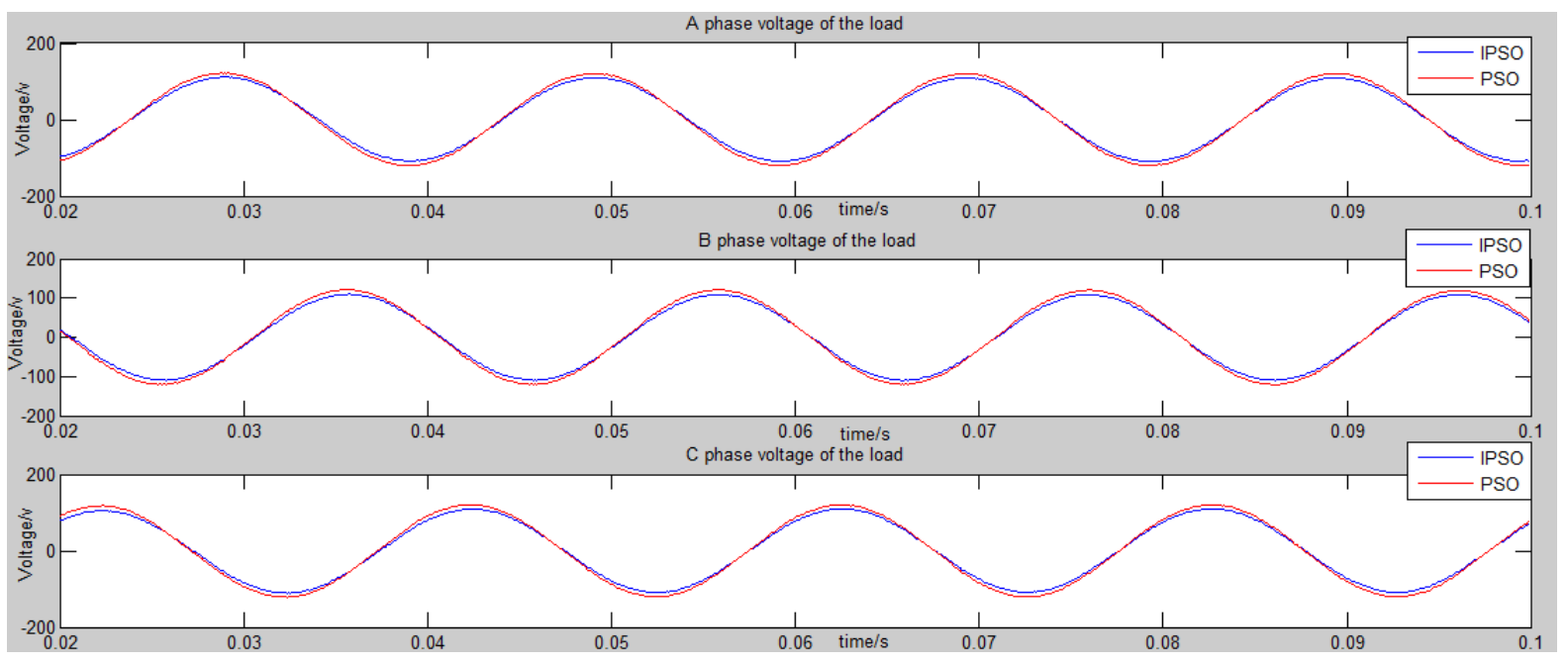

Figure 5. The load output voltage comparison chart of IPSO and PSO optimization from islanding to Grid-connected operation with one micro-source.

filter parameters can effectively reduce output voltage harmonic ratio of the load side from grid-connected operation to islanding operation whether in a multi-sources or single-source situation.

Similarly, according to the Figure 3 of the comparison of A, B, C three-phase voltage of the simulation results by using IPSO to optimize the filter parameters and A, B, C three-phase voltage of the simulation results by using PSO, we can find that the load side output voltage harmonic rate decreases from $5.96 \%$ to $4.97 \%$. And according to the Figure 5 of the comparison of A, B, C three-phase voltage of the simulation results by using IPSO and A, B, C three-phase voltage of the simulation results by using PSO, we can find that the load side output voltage harmonic rate decreases from $6.16 \%$ to $5.10 \%$. Therefore, using IPSO to optimize filter parameters can effectively reduce output voltage harmonic ratio of the load side from islanding operation to grid-connected operation whether in a multi-sources or single- source situation.

\section{Conclusion}

In this paper, we proposed a method that using immune particle swarm algorithm to optimize the filter parameters of micro-grid system to solve the problem of harmonics generating by micro-grid inverters and other power electronic equipment in the micro-grid system. Immune particle swarm algorithm can effectively avoid the pre- 
mature convergence and improve the convergence rate. Through case studies, according to the comparison of optimization parameters and objective function value with the PSO and IPSO, we can know that IPSO for the optimization of micro-grid system filter parameters and reducing the load on the output terminal voltage harmonic ratio is effective and feasible.

\section{References}

[1] Zhao, H.-W. and Wu, T.-T. (2008) Review of Distributed Generation Based Micro-Grid Technology. Proceedings of the CSU-EPSA, 20, 121-126.

[2] Villeneuve, P.L. (2004) Concerns Generated by Islanding. IEEE Power and Energy Magazine, 2, 49-53.

[3] Wang, C.-S., Xiao, Z.-X. and Wang, S.-X. (2008) Integrated Control and Analysis of Micro-Grid. Automation of Electric Power Systems, 32, 98-103.

[4] (2013) Harmonics Suppression of Current Transformer Methods and Countermeasures in Micro-Grid. http://www.papershome.com/gongxue/dianli/15168_2.html

[5] Li, F.-X. and Zhao, B.-H. (2001) The Study and Apply of Technology about Harmonic Restraining. Proceedings of the CSU-EPSA, 13, 5.

[6] Li, Y., Vilathgamuwa, D.M. and Loh, P.C. (2005) Micro-Grid Power Quality Enhancement Using a Three-Phase Four Wire Grid-Interfacing Compensator. IEEE Transactions on Industry Applications, 41, 1707-1719. http://dx.doi.org/10.1109/TIA.2005.858262

[7] Zhang, W. (2006) Reactive Power Optimization Research of Power System Based on Particle Swarm Algorithm. Shandong University.

[8] Yan, J., Hu, T.S. and Huang, C.H. (2011) An Improved Particle Swarm Optimization Algorithm. Applied Mathematics and Computation, 37, 1232-1247.

[9] Xiong, H.-G., Chang, H.-Z. and Li, H.-Z. (2006) Multi-Objective Reactive Power Optimization Based on Immune Algorithm. Proceedings of the CSEE, 104-108.

[10] Liu, K.-Y., Sheng, W.-X. and Li, Y.-H. (2007) ReactiVe Power Optimization Based on Improved Immunity Genetic Algorithm. Power System Technology, 31, 11-16.

[11] Mohamed, Y.A.-R.I. and El-Saadany, E.F. (2008) Adaptive Decentralized Droop Controller to Preserve Power Sharing Stability of Paralleled Inverters in Distributed Generation Microgrids Power Electronics. IEEE Transactions, 28062816.

[12] Han, L. (2008) The Research and Application of Immunity Swarm Algorithm. Xi'an University of Engineering, Xi'an.

[13] Nie, R. and Yue, J.-H. (2009) Seismic Wave Impedance Inversion Based Onadaptive Immune Clonal Particles Swarm Optization. Computer Applications and Software. 